\title{
Non-Contact Sleep Staging Algorithm Based on Physiological Signal Monitoring
}

\author{
Jian He, Bo Han \\ Faculty of Information Technology, Beijing University of Technology, Beijing 100124, China
}

Keywords: EEG, sleep monitoring, sleep segmentation, detection system

\begin{abstract}
Sleep is an important physiological activity of human body, in recent years, the sleep quality has been highlighted; therefore, the paper explores the effective and feasible method to monitor the sleep quality from the perspective of EEG, and designs a portable sleep quality monitoring system from the perspective of software and hardware. Besides, it studies and analyzes the data collected based on the portable EEG acquisition equipment as well as the CAP Sleep EEG database, and explores the effective algorithm for sleep segmentation by aid of the power spectrum analysis and BP neural network. In addition, the experiment conducts evaluation on the sleep segmentation and quality preliminarily, and proves the correctness of the portable sleep quality monitoring system as well as the effectiveness of implementing the sleep segmentation based on the EEG data. The research puts forward to rely on the single-guide EEG for implementing the sleep segmentation, which provides important experience reference for researching the portable and market-oriented sleep monitoring equipment as well as other applications.
\end{abstract}

\section{Introduction}

Sleep is an important physiological activity of human body. From the cradle to the grave, humans usually spend the whole life in sleep and wakefulness. In addition, humans can allay tiredness to recover the spirit and physical power better through high-quality sleep, which can make humans keep good wakefulness situation and improve their working and learning efficiency. Humans spend $1 / 3$ of the lifetime for sleep. However, we have known little about this kind of physiological phenomenon, and have conducted scientific researches on sleep for only several decades. In 1987, Lomis, Harvey and Hobart noticed that sleep was not in a stable situation but a series of organized and periodical change. In 1986, Rechtschaffen et al. reaffirmed the sleep segmentation criterion made by Dement and Kleitma, and made some modifications and supplements based on the ten-year experience thus to make it more perfect. In 2007, the American Academy of Sleep Medicine (AASM) made improvement based on the above criterion and published the new and professional sleep segmentation criterion, which specifies the specific collection criterion and judgement methods of various indicators.

\section{Sleep quality monitoring methods based on EEG}

\subsection{Preprocessing method for EEG}

EEG belongs to the non-stationary random signal, in the experiment, the EEG collected is singleguided, therefore, the signal is not stable with serious noise. Therefore, the preliminary low-pass digital filtering pre-processing is needed. As a matter of convenience, the upper limit of the frequency attenuation band should be $50 \mathrm{~Hz}$ which is higher than the effective frequency of $30 \mathrm{~Hz}$ in the EEG analysis.

The digital filter includes the finite impulse response (FIR) filter and the infinite impulse response (IIR) filter. Wherein, the FIR filter can obtain strict linear phase, which needs to adopt higher order (five to ten times higher than IIR) compared with IIR and is featured with convenient software. [10]

Provided that the unit impulsion response h(n) of the FIR filter is represented by a sequence with the length of $\mathrm{N}$, and then the system function of the filter will be: 


$$
H(z)=\sum_{n=0}^{N-1} h(n) z^{-n}
$$

The difference form of the above formula is:

$$
y(n)=\sum_{m=0}^{N-1} h(m) x(n-m)
$$

Because the ideal filter is not continuous in the boundary frequency, its time-domain signal hd(n) is certain to be the infinite time width. Therefore, it is needed to capture the filter curve featured with ideal linear phase with window function:

$$
h(n)=h_{d}(n) w(n)
$$

This kind of design idea is called as window function design method.

Wherein, the common Hamming Window function is as follows:

$$
w_{H m}(n)=\left[0.54-0.46 \cos \frac{2 \pi n}{N-1}\right] R_{N}(n)
$$

The amplitude value function:

$$
W_{H m}(\omega)=0.54 W_{R}(\omega)+0.23 W_{R}\left(\omega-\frac{2 \pi}{N-1}\right)+0.23 W_{R}\left(\omega+\frac{2 \pi}{N-1}\right)
$$

Design the 300-point FIR low-pass filter by aid of the firl tool of Matlab, adopt Hamming Window, take $512 \mathrm{~Hz}$ and $50 \mathrm{~Hz}$ as the sampling frequency and attenuation band respectively, and then get the following amplitude-frequency response of the filter:
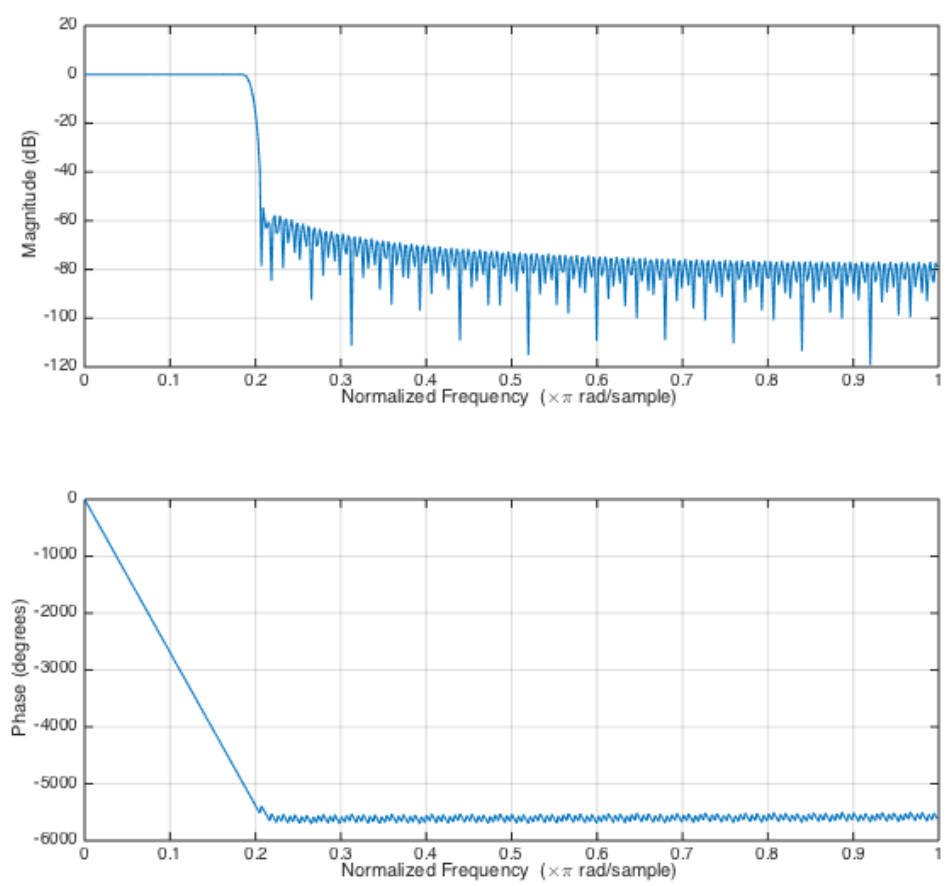

Figure 1 Amplitude-frequency response of the 300-point low-pass filter

(Adopt the normalized angular frequency of $2 \pi$, that is $512 \mathrm{~Hz}$ of the actual sampling frequency)

\subsection{Frequency-domain analysis on EEG}

From the perspective of frequency from high to low, EEG can be divided into: $\beta$ wave $(14 \sim 30 \mathrm{~Hz}), \alpha$ wave $(8 \sim 14 \mathrm{~Hz}), \theta$ wave $(4 \sim 8 \mathrm{~Hz}), \delta$ wave $(0.5 \sim 4 \mathrm{~Hz})$. 
Table 1 Frequency band division of EEG and the mental state of brain reflected from different types of EEG

\begin{tabular}{|l|l|l|}
\hline EEG & Frequency range & Mental state \\
\hline$\beta$ wave & $14 \sim 30 \mathrm{~Hz}$ & $\begin{array}{l}\text { The movement perception is rhythmical and the concentration } \\
\text { can be focused in relaxed state, coordinated and thoughtful, } \\
\text { alerted in self and surrounding environment, excited }\end{array}$ \\
\hline$\alpha$ wave & $8 \sim 14 \mathrm{~Hz}$ & Relaxed without tiredness, calm and conscious \\
\hline$\theta$ wave & $4 \sim 8 \mathrm{~Hz}$ & Intuitive, reminiscent, visionary, imaginative, light sleep \\
\hline$\delta$ wave & $0.5 \sim 4 \mathrm{~Hz}$ & Deep sleep, non-rapid-eye movement sleep, unconscious \\
\hline
\end{tabular}

The Fourier Transform of the discrete-time series $\mathrm{x}(\mathrm{n})$ is:

$$
F(\omega)=\sum_{n=1}^{N} x(n) e^{-j \omega n}
$$

If the auto-correlation function $\mathrm{r}(\mathrm{k})$ of the random signal $\mathrm{x}(\mathrm{n})$ is known, the power spectral density function will be:

$$
P(\omega)=\sum_{k=-\infty}^{+\infty} r(k) e^{-j \omega k}
$$

Besides, the power spectral function can also be:

$$
P(\omega)=\lim _{N \rightarrow \infty} E\left[\frac{1}{N}\left|\sum_{n=1}^{N} x(n) e^{-j \omega n}\right|^{2}-\right.
$$

Theoretically, the discrete signal processing method can conduct correct analysis on the signal of limited-bandwidth, while the signal of limited-bandwidth is infinitely long in time domain, therefore, conducting Fourier Transform on one certain section is equal to adding rectangular window calculation on the original signal. The windowing plays the smooth role in the original power spectral from the perspective of frequency domain.

$$
\hat{P}(\omega)=\frac{1}{N}\left|\sum_{n=1}^{N} w(n) x(n) e^{-j \omega n}\right|^{z}
$$

Wherein, w(n) represents the window function. The common windows involve the quarter window, Hanning Window, Hamming window and Blackman window, the side lobe level and resolution rate of which is lower than those of rectangle window. Here is the Hamming window.

\subsection{Improvement algorithm for sleep segmentation judgement}

In accordance with the 2007 criterion of AASM, the BBG criteria of sleep segmentation are as follows: 
Table 2 BBG criteria of sleep segmentation

\begin{tabular}{|l|l|}
\hline Stages of sleep & $\begin{array}{l}\text { Division rule (only EEG is contained, and the exceptional } \\
\text { circumstance can be neglected ) }\end{array}$ \\
\hline W (wakefulness) & The $\alpha$ wave content in the occipital region is larger than $50 \%$ \\
\hline N2 (non-rapid eye movement 1) & $\begin{array}{l}\alpha \text { wave is weak, and the } 4 \sim 7 \mathrm{~Hz} \text { low-amplitude wave content is larger } \\
\text { than 50\% }\end{array}$ \\
\hline N3 (non-rapid eye movement 3) & $\begin{array}{l}\text { The initial stage: the K complex wave or spindle wave irrelevant to } \\
\text { wakefulness appears } \\
\text { Continuous stage: the } 4 \sim 7 \mathrm{~Hz} \text { low-amplitude wave content } \\
\text { (excluding the K complex wave or spindle wave ) }\end{array}$ \\
\hline R (rapid eye movement) & $\begin{array}{l}\text { Appear with the } 4 \sim 7 \mathrm{~Hz} \text { low-amplitude wave (excluding the } \mathrm{K} \\
\text { complex wave or spindle wave) }\end{array}$ \\
\hline
\end{tabular}

Wherein, the depth of sleep in the stage of non-rapid eye movement: N3, N2, N1 successively from deep to light.

The Artificial Neural Network is the complex network composed of numerous simple processing units based on wide connection, which is used for simulating the structure and behavior of the brain's neural network. It reflects many basic features of human brain function, however, it is not the real reflection of human brain completely but some kind of simplification, abstract and simulation to human brain. In the various learning algorithms, the back propagation algorithm based on multi-layer network (BP algorithm in short) is the most widely applied. BP algorithm was firstly raised by Werbos in 1974, which was developed by Rumelhart et al. in 1985, who also put forward clear and strict algorithm. Besides, BP algorithm is suitable to the forward network and adopts the tutor-based training form, which provides the input vector set as well as the output vector set and adjusts the connection weight of network through back-propagation learning algorithm thus to make the network output close to the expected output under the significance of minimum mean squared error, in addition, the process of backward learning is composed of forward propagation and back propagation. In the process of forward propagation, the input information is processed by hidden neuron on a layer-on-layer basis and is transmitted to the output layer, if the output layer can not realize the expected output, turn to the back propagation, return the error between the actual output and the expected output along the original connection channel, and modify the connection weight of the different-layer neurons thus to reduce the error and turn to the forward propagation for repeated circulation until the error is lesser than the given value.

\section{Analysis on experiment data}

\subsection{Effect verification of the filter}

In order to verify the effect of the filter and the hardware performance, capture a 4-second length of signal in the collected EEG under the situation of wakefulness for verification. Through detection, the effect of the filter is good, and the data based on real-time filtering has been proved to conform to the requirements of EEG analysis. 

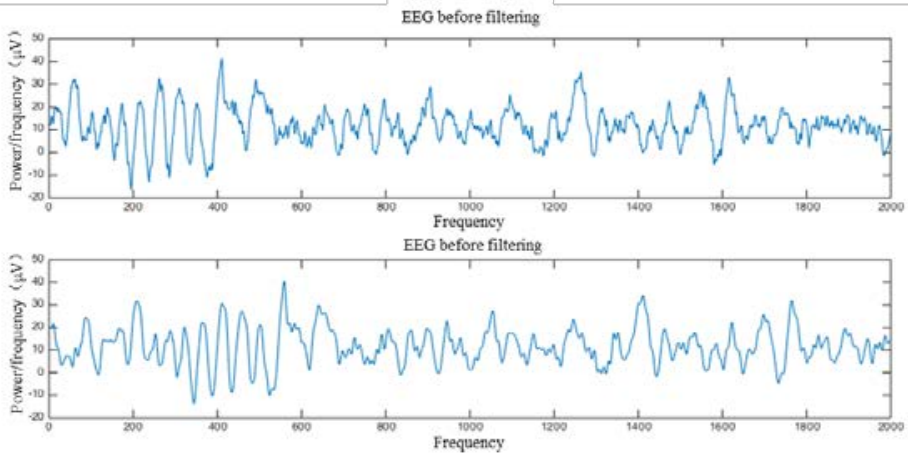

Figure 2 Comparison of the EEG waveform before and after filtering (the sampling frequency of $512 \mathrm{~Hz})$
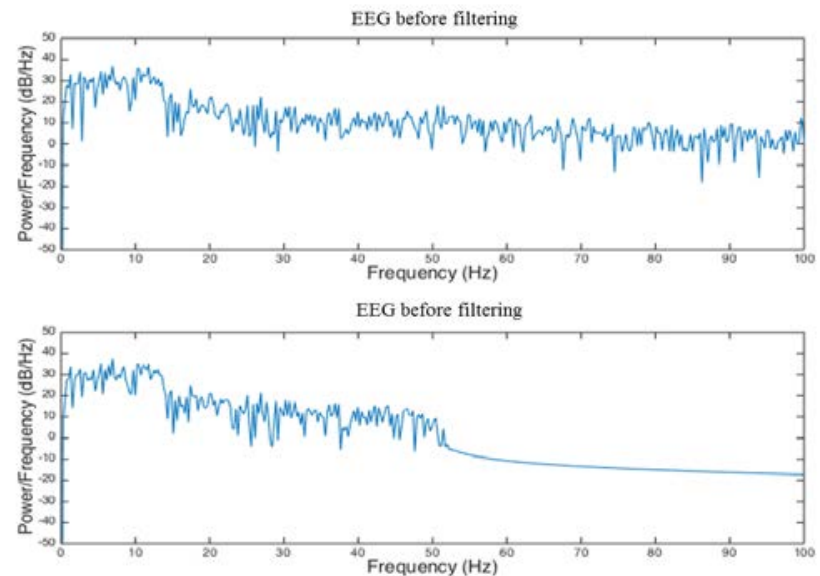

Figure 3 Comparison of the EEG frequency spectrum before and after filtering (the sampling frequency of $512 \mathrm{~Hz}$ )

\subsection{Effect verification of windowing frequency domain analysis}

Because the power proportion of the low-frequency EEG will increase and decrease under the situations of deep sleep and light sleep respectively, the power proportion of the single-frequency wave can be used for determining the depth of sleep simply.

When the experiment objects wear the EEG acquisition equipment for 9 hours at night, analyze the power proportion of $\delta$ wave $(0.5 \sim 4 \mathrm{~Hz})$ based on the Hamming window frequency domain every 30 -second data and get the following figure. Though comparison and reference, the figure can roughly reflect the depth of sleep, which conforms to the objective rule of sleep cycle. The effect of the windowing frequency domain analysis can be verified.

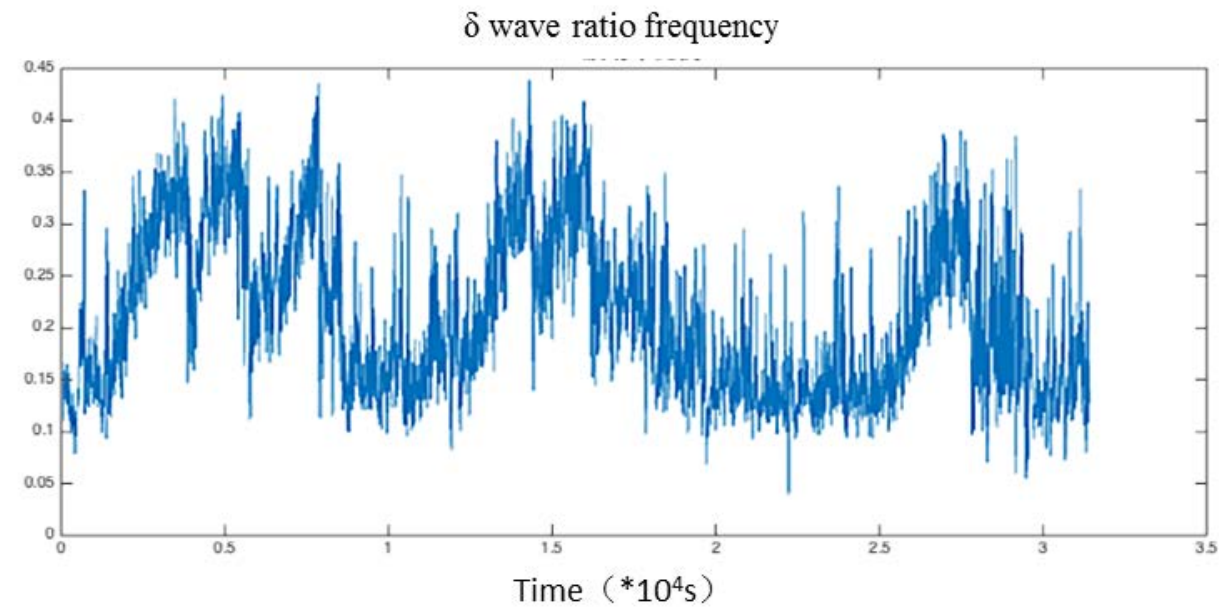

Figure 4 The power proportion of $\delta$ wave based on windowing frequency domain analysis 


\subsection{Judgement method of sleep segmentation}

Under the situation that the experiment objects wear the EEG acquisition equipment without taking any ancillary drugs or intervention treatment for continuous five nights, collect the EEG. Wherein, the experiment objects are healthy, who have normal and regular sleep time with complete sleep cycle. Owing to the "first-night effect" of the sleep monitoring experiment, namely the experiment objects are difficult to sleep, or are easily roused from sleep without complete sleep cycle when they firstly wear the equipment, therefore, adopt the data of the second to fifth night for analysis. The sampling frequency is fixed in $512 \mathrm{~Hz}$ and the continuous sleep time every night exceeds 6 hours. Select the length of ten minutes (600 seconds) and get the power proportion of the EEG in different frequency bands based on the Hamming window frequency domain analysis every 30 seconds. The following rule can be found: the summits or troughs of $\delta$ wave and $\alpha$ wave frequencies are fused, namely when the frequency of one kind of wave is in the summit, the frequency of another kind of wave is in the trough. The generality of the rule in the experiment can be confirmed after analyzing the non-continuous collection time for few decades.

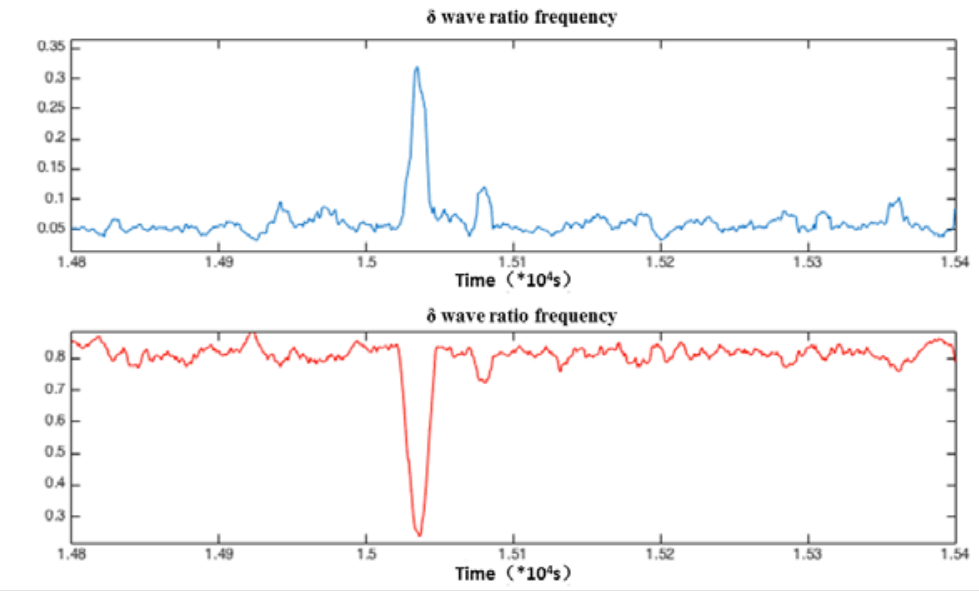

Figure 5 The summits or troughs of $\delta$ wave and $\alpha$ wave frequencies are fused

\section{Conclusions}

The experiment proves that the portable EEG acquisition equipment is featured with incomparable movability compared with the traditional equipment, which is suitable for personal and family use; high-quality EEG can be obtained for analysis based on it, and reliable monitoring and evaluation on sleep quality can be conducted combined with the neural network algorithm thus to realize the sleep segmentation preliminarily. However, it is needed to collect the multi-guided EEG as well as other ECG and breathing signals for comprehensive evaluation based on the strict sleep segmentation criteria, besides, higher professional skills and quality are needed because the portable sleep monitoring system cannot meet the requirements. The experiment result provides significant experiment basis and reference for people to better research the portable sleep monitoring system. The exploration on the relationship between the sing-guided EEG and sleep as well as the modern advanced analysis algorithm of EEG are the important theoretical basis for the experiment. In addition, the explorations on the induction and treatment theory of EEG, the correctness and reliability of the portable EEG acquisition equipment as well as the marketorientated diagnosis and evaluation can also be taken as the development direction of the paper.

\section{References}

[1] Jennifer W. Chan, Yingyue Zhang, and Kathryn E. Uhrich, Amphiphilic Macromolecule SelfAssembled Monolayers Suppress Smooth Muscle Cell Proliferation, Bioconjugate Chemistry, 2015, 26(7), 1359-1369.

[2] Jennifer W. Chan, Yingyue Zhang, and Kathryn E. Uhrich, Amphiphilic Macromolecule Self- 
Assembled Monolayers Suppress Smooth Muscle Cell Proliferation, Bioconjugate Chemistry, 2015, 26(7), 1359-1369.

[3] Kurup, P.; Sullivan, C.; Hannagan, R.; Yu, S.; Azimi, H.; Robertson, S.; Ryan, D.; Nagarajan, R.; Ponrathnam, T.; Howe, G. A Review of Technologies for Characterization of Heavy Metal Contaminants. Indian Geotech J 2017, 47 (4), 421-436.

[4] Ghebrebrhan, M.; Aranda, F.; Walsh, G.; Ziegler, D.; Giardini, S.; Carlson, J.; Kimball, B.; Steeves, D.; Xia, Z.; Yu, S.; et al. Textile Frequency Selective Surface. IEEE Microwave and Wireless Components Letters 2017, 27 (11), 989-991.

[5] Malarkodi, M.P., Arunkumar, N., Venkataraman, V. Gabor wavelet based approach for face recognition (2013) International Journal of Applied Engineering Research, 8 (15), pp. 1831-1840. 